$11-1-2002$

\title{
On Adaptive Wavelet Estimation of a Derivative and Other Related Linear Inverse Problems
}

T. Tony Cai

University of Pennsylvania

Follow this and additional works at: https://repository.upenn.edu/statistics_papers

Part of the Applied Mathematics Commons, Physics Commons, and the Statistics and Probability Commons

\section{Recommended Citation}

Cai, T. (2002). On Adaptive Wavelet Estimation of a Derivative and Other Related Linear Inverse Problems. Journal of Statistical Planning and Inference, 108 (1-2), 329-349. http://dx.doi.org/10.1016/ S0378-3758(02)00316-6

This paper is posted at ScholarlyCommons. https://repository.upenn.edu/statistics_papers/580

For more information, please contact repository@pobox.upenn.edu. 


\title{
On Adaptive Wavelet Estimation of a Derivative and Other Related Linear Inverse Problems
}

\begin{abstract}
We consider a block thresholding and vaguelet-wavelet approach to certain statistical linear inverse problems. Based on an oracle inequality, an adaptive block thresholding estimator for linear inverse problems is proposed and the asymptotic properties of the estimator are investigated. It is shown that the estimator enjoys a higher degree of adaptivity than the standard term-by-term thresholding methods; it attains the exact optimal rates of convergence over a range of Besov classes. The problem of estimating a derivative is considered in more detail as a test for the general estimation procedure. We show that the derivative estimator is spatially adaptive; it automatically adapts to the local smoothness of the function and attains the local adaptive minimax rate for estimating a derivative at a point.
\end{abstract}

\section{Keywords}

block thresholding, derivative, linear inverse problems, vaguelets, wavelets

Disciplines

Applied Mathematics | Physics | Statistics and Probability 


\title{
ON ADAPTIVE WAVELET ESTIMATION OF A DERIVATIVE AND OTHER RELATED \\ LINEAR INVERSE PROBLEMS
}

\author{
by \\ T. Tony Cai \\ Purdue University \\ Technical Report \#99-07
}

\author{
Department of Statistics \\ Purdue University \\ West Lafayette, IN 47907-1399 \\ USA
}

May 1999 


\title{
On Adaptive Wavelet Estimation of a Derivative And Other Related Linear Inverse Problems
}

\author{
T. Tony Cai \\ Department of Statistics \\ Purdue University
}

\begin{abstract}
We consider a block thresholding and vaguelet-wavelet approach to certain statistical linear inverse problems. Based on an oracle inequality, an adaptive block thresholding estimator for linear inverse problems is proposed and the asymptotic properties of the estimator are investigated. It is shown that the estimator enjoys a higher degree of adaptivity than the standard term-by-term thresholding methods; it attains the exact optimal rates of convergence over a range of Besov classes. The problem of estimating a derivative is considered in more detail as a test for the general estimation procedure. We show that the derivative estimator is spatially adaptive; it automatically adapts to the local smoothness of the function and attains the local adaptive minimax rate for estimating a derivative at a point.
\end{abstract}

Keywords: Adaptivity; Block thresholding; Derivative; James-Stein estimator; Linear inverse problems; Minimax; Nonparametric regression; Vaguelets; Wavelets. 


\section{Introduction}

Statistical linear inverse problems pertain to situations where one is interested in estimating an unknown object $f(t)$ based on noisy observations on $(K f)(t)$, where $K$ is a linear operator. Such problems arise in many scientific settings, from medical imaging to astronomy. Suppose we observe

$$
d Y(t)=(K f)(t) d t+d W(t)
$$

where $W(t)$ is Brownian motion. Examples of the operator $K$ in (1) include integration, fractional integration, convolution, and Radon transform. We are interested in estimating the function $f$ from the data $Y$ and we measure the estimation accuracy by the mean integrated square error:

$$
R(\hat{f}, f)=E\|\hat{f}-f\|_{2}^{2}
$$

Traditional methods usually use regularization and the Singular Value Decomposition (SVD). See, e.g., Tikhonov \& Arsenin (1977), O'Sullivan (1986), and Johnstone \& Silverman (1990). The SVD method expands the function $f$ in a basis formed by the eigenfunctions of the self-adjoint operator $K^{*} K$ where $K^{*}$ is the adjoint of $K$. When noisy data about $(K f)(t)$ are observed, the series is truncated and the coefficients of the eigenfunctions in the expansion are estimated from the data. Johnstone \& Silverman (1990) showed that a properly tuned SVD estimator attains the minimax rate of convergence over some homogeneous function classes. The SVD method has certain limitations, however. The basis functions are completely derived from the operator $K$, not from the object of interest $f$. When the function $f$ is of inhomogeneous smoothness, the representation of $f$ by the eigenfunctions of $K^{*} K$ is often inefficient and the resulting estimator does not perform well.

Wavelet bases offer efficient representations for functions in a wide range of function spaces and wavelet methods have demonstrated considerable success in nonparametric function estimation in terms of spatial adaptivity and asymptotic optimality. A properly chosen wavelet basis can simultaneously quasi-diagonalize both the operator $K$ and the functions in a range of function classes. Donoho (1995) proposed the Wavelet-Vaguelet Decomposition (WVD) method for linear inverse problems which works by expanding the function $f$ in a wavelet series and producing a corresponding vaguelet series for $K f$, and then estimating the wavelet coefficients by thresholding the empirical vaguelet coefficients. Donoho (1995) showed that the estimator with optimal threshold attains the minimax rate of convergence. Johnstone (1999) proposed a specific thresholding rule and showed that the resulting estimator is adaptive and rate-optimal.

Abramovich and Silverman (1998) took another wavelet approach. They introduced the Vaguelet-Wavelet Decomposition (VWD) method which first expands $K f$ in a wavelet series, then thresholds the noisy empirical wavelet coefficients and finally maps back by $K^{-1}$ to obtain an estimator of $f$ in terms of a vaguelet series. The VWD estimator is a method of presmoothing the estimator. Abramovich and Silverman (1998) used a standard term-by-term thresholding method for estimating the wavelet coefficients of $K f$ and it is shown that the resulting VWD estimator is within a logarithmic factor of the minimax risk. 
The VWD approach is conceptually attractive. However, the term-by-term thresholding method used in estimating the wavelet coefficients of $K f$ has drawbacks. The difficulty of term-by-term thresholding is caused by the relative inaccuracy in estimating the individual wavelet coefficients. As a result, it creates a logarithmic penalty in the mean squared error. The problem can not be solved by simply fine tuning the universal threshold level.

Cai (1999) considered a local block thresholding rule, based on an oracle inequality, for wavelet function estimation in the context of nonprametric regression and white noise model. The estimator thresholds the empirical wavelet coefficients in groups rather than individually, making simultaneous decisions to retain or to discard all the coefficients within a block. The aim is to increase estimation accuracy by utilizing information about neighboring wavelet coefficients. As shown in Cai (1999) the block thresholding estimator achieves simultaneously three objectives: adaptivity, spatial adaptivity, and computational efficiency. The estimator enjoys a higher degree of adaptivity than the standard term-by-term thresholding methods. Other block thresholding rules have been considered by Hall et al. (1999) and Cai \& Silverman (1999). In the present paper, we demonstrate that the approach of block thresholding can be used for linear inverse problems as well.

We first briefly review the WVD approach of Donoho (1995) and Johnstone (1999) and the VWD approach of Abramovich and Silverman (1998) in Section 2. After Section 3.1 in which block thresholding method is introduced, we present in Section 3.2 an estimator for linear inverse problems using the vaguelet-wavelet decomposition which incorporates the block thresholding approach in Cai (1999). Here, the wavelet coefficients of $K f$ are divided into blocks and coefficients within a block are estimated simultaneously. The threshold is based on the block projection oracle inequality developed in Cai (1999). The asymptotic properties of the estimator are investigated. We show in Section 4 that the estimator enjoys a high degree of adaptivity. Specifically, we prove that the estimator simultaneously attains the exact optimal rate of convergence over a range of the Besov classes with $p \geq 2$ without prior knowledge of the smoothness of the underlying functions. Over the Besov classes with $p<2$, the estimator simultaneously achieves the optimal convergence rate within a logarithmic factor.

We consider in Section 5 the problem of estimating the derivative of a function $g$ as a test of our estimation procedure. This problem fits into the framework of (1) by setting $K$ to be the integration operator. It is an important estimation problem. For example, in growth studies the derivative of height or weight is important in determining growth spurts and times at which height or weight are changing rapidly (see Gasser et al. (1984)). We study the local adaptivity of the estimator and the numerical implementation of the procedure. We show that the estimator is spatially adaptive; it attains the local adaptive minimax rate for estimating a derivative at a point. The block thresholding method discussed in the present paper can be extended and generalized in various ways. Section 6 discusses some variations of the method. All the proofs are contained in Section 7. 


\section{WVD and VWD}

Wavelet series are generated from dilations and translations of a special function, called the mother wavelet $\psi: \psi_{j, k}(t)=2^{j / 2} \psi\left(2^{j} t-k\right)$. The collection $\left\{\psi_{j, k}: j, k \in Z\right\}$ forms an orthonormal basis in $L_{2}(\mathbb{R})$. Wavelets are well localized and offer efficient representations for functions in a wide range of function spaces. See Meyer (1992) for further details on data compression and localization properties of wavelets. The mother wavelet can be chosen to be compactly supported. We will always use compactly supported wavelets in the present paper.

We call a wavelet $\psi$ r-regular if $\psi$ has $r$ continuous derivatives and vanishing moments up to order $r$, i.e., $\int t^{\ell} \psi(t) d t=0$ for $\ell=0,1, \cdots, r$. For a given mother wavelet $\psi$ there is an associated father wavelet $\phi$. The father wavelet is also localized with $\int \phi(t) d t=1$ and has the same degree of smoothness as $\psi$. Furthermore, the father wavelet $\phi$ can be chosen to have vanishing moments, $\int t^{\ell} \phi(t) d t=0$ for $\ell=1, \cdots, r$. Such wavelets are called coiflets (see Daubechies (1992)). The dilations and translations of the father wavelet $\left\{\phi_{l, k}(t)=2^{l / 2} \phi\left(2^{l} t-k\right), k \in Z\right\}$ together with $\left\{\psi_{j, k}: j \geq l, k \in Z\right\}$ form an inhomogeneous orthonormal wavelet basis; see, e.g., Daubechies (1992).

An orthonormal wavelet basis has an associated orthogonal Discrete Wavelet Transform (DWT) that is norm-preserving and transforms sampled data into the wavelet coefficient domain. See Daubechies (1992) and Strang (1992) for more on the wavelets and the discrete wavelet transform.

Vaguelets are closely associated with wavelets. Like wavelets, vaguelets are localized and oscillating; and vaguelets are "almost" orthogonal. Vaguelets are indexed in the same way as the wavelets. For example, if $\psi$ is a compactly supported mother wavelet and is sufficiently smooth, then $\left\{u_{j, k}(t)=2^{j / 2} \psi^{\prime}\left(2^{j} t-k\right) j, k \in Z\right\}$ constitutes a vaguelet system. In particular, there exists some constant $C>0$ such that

$$
\left\|\sum_{j, k} a_{j, k} u_{j, k}(t)\right\|_{2} \leq C\left\|\left(a_{j, k}\right)\right\|_{\ell_{2}}
$$

for every sequence $\left(a_{j, k}\right)$. Such a sequence $\left\{u_{j, k}\right\}$ satisfying (3) is called a Bessel sequence (see Young (1976)). The readers are referred to Meyer \& Coifman (1997, pp. 56) for the formal definition of vaguelets. See also Donoho (1995).

\subsection{Wavelet-Vaguelet Decomposition}

Donoho (1995) showed that, when the orthonormal wavelet basis $\left(\psi_{j, k}\right)$ is properly chosen, for a special class of operators $K$ there exist two associated biorthogonal vaguelet systems $\left(u_{j, k}\right)$ and $\left(v_{j, k}\right)$ satisfying the following.

1. Quasi-singular value relations

$$
K \psi_{j, k}=r_{j} v_{j, k}, \quad K^{*} u_{j, k}=r_{j} \psi_{j, k} .
$$

with quasi-singular values $\left(r_{j}\right)$, depending on the resolution level $j$ but not the spatial index $k$. 
2. Biorthogonality relations

$$
\left\langle u_{j, k}, v_{l, m}\right\rangle=\delta_{j, l} \delta_{k, m}
$$

3. Near-orthogonality relations

$$
\begin{aligned}
& b\left\|\left(a_{j, k}\right)\right\|_{\ell_{2}} \leq\left\|\sum_{j, k} a_{j, k} u_{j, k}\right\|_{2} \leq B\left\|\left(a_{j, k}\right)\right\|_{\ell_{2}} \\
& b\left\|\left(a_{j, k}\right)\right\|_{\ell_{2}} \leq\left\|\sum_{j, k} a_{j, k} v_{j, k}\right\|_{2} \leq B\left\|\left(a_{j, k}\right)\right\|_{\ell_{2}}
\end{aligned}
$$

for every sequence $\left(a_{j, k}\right)$ where $B>b>0$ are some fixed constants.

When this decomposition exists, the function $f$ can be represented as a wavelet series and correspondingly expands $K f$ in a vaguelet series:

$$
f=\sum_{j, k}\left\langle f, \psi_{j, k}\right\rangle \psi_{j, k}, \quad \text { and } \quad K f=\sum_{j, k}\left\langle f, \psi_{j, k}\right\rangle r_{j} v_{j, k} .
$$

The wavelet coefficients of $f$ can be reproduced from $K f:\left\langle f, \psi_{j, k}\right\rangle=\left\langle K f, u_{j, k}\right\rangle r_{j}^{-1}$. This yields $K f=\sum_{j, k}\left\langle K f, u_{j, k}\right\rangle v_{j, k}$ and the following representation for $f$ :

$$
f=\sum_{j, k}\left\langle K f, u_{j, k}\right\rangle r_{j}^{-1} \psi_{j, k}
$$

It is clear that only special operators $K$ satisfy (4)-(7). For example, the conditions hold for homogeneous operators, which, satisfy $K[f(a t)]=a^{-\gamma}(K f)(a t)$ for some constant $\gamma$, called the index of the operator. Examples of homogeneous operators include integration, fractional integration and, in the two-dimensional case, the Radon transform. For homogeneous operators with index $\gamma, r_{j}$ in (4) equals $C_{K} 2^{-j \gamma}$ where $C_{K}$ is a constant. The properties 4)-(7) also hold for various convolution operators (see Donoho (1995) and Johnstone (1999)).

Based on the representation (8), the problem of estimating $f$ from noisy observations of $K f$ is now transformed into the problem of estimating the vaguelet coefficients $\zeta_{j, k}=$ $\left\langle K f, u_{j, k}\right\rangle$. Suppose we observe $Y(t)$ as in (1). We can form the empirical vaguelet coefficients $b_{j, k}=\int u_{j, k}(t) d Y(t)$ and decompose it as

$$
b_{j, k}=\zeta_{j, k}+\epsilon_{j, k}
$$

where $\epsilon_{j, k}=\int u_{j, k}(t) d W(t)$ are the vaguelet coefficients of a Brownian motion. The $\epsilon_{j, k}$ are normally distributed, but they are not independent since the vaguelets $u_{j, k}$ are not orthogonal. One can then apply a term-by-term thresholding rule to the empirical vaguelet coefficients to obtain an estimate of the true vaguelet coefficients.

$$
\hat{\zeta}_{j, k}=\eta_{\lambda}\left(b_{j, k}\right)
$$

where $\eta_{\lambda}(\cdot)$ can be either the soft threshold function

$$
\eta_{\lambda}^{s}(x)=\operatorname{sgn}(x)(|x|-\lambda)_{+}
$$


or the hard threshold function

$$
\eta_{\lambda}^{h}(x)=x I(|x|>\lambda) .
$$

The wavelet-vaguelet decomposition estimator $\hat{f}_{W V D}$ is given by

$$
\hat{f}_{W V D}=\sum_{j, k} \hat{\zeta}_{j, k} r_{j}^{-1} \psi_{j, k} .
$$

Donoho (1995) showed that, if the threshold $\lambda$ is optimally chosen level by level, the WVD estimator attains the minimax rate. However, no specific rate-optimal WVD estimator is provided in the paper.

Johnstone (1999) proposes a thresholding rule for estimating $\zeta_{j, k}$ based on the Stein's unbiased risk estimate (SURE). At each resolution level $j$, the threshold $\lambda_{j}$ is empirically chosen to be the minimizer of the Stein's unbiased risk estimate. The resulting SURE estimator is shown to be adaptive and attains the minimax rate of convergence over a range of Besov classes.

\subsection{Vaguelet-Wavelet Decomposition}

Abramovich \& Silverman (1998) introduced an alternative method, called the vagueletwavelet decomposition (VWD), which expands $K f$ rather than $f$ in a wavelet series. The method thresholds the wavelet coefficients of the observed data $Y$ to obtain an estimate of the wavelet expansion of $K f$ and then maps back by $K^{-1}$ to obtain an estimate of $f$ in terms of a vaguelet series. The vaguelet-wavelet decomposition approach can be regarded as a plug-in estimator or a presmoothing estimator. Here we first construct a wavelet estimator of $K f$ and then apply $K^{-1}$ to obtain an estimate $f$ itself. The method is straightforward and can be formally described as follows.

Assume the existence of constants $\beta_{j}$ such that (7) holds for $w_{j, k}=K^{-1} \psi_{j, k} / \beta_{j}$. If $K$ is homogeneous of index $\gamma$ then $\beta_{j}$ is proportional to $2^{\gamma j}$. The function $f$ can then be written as

$$
f=\sum_{j, k}\left\langle K f, \psi_{j, k}\right\rangle \beta_{j} w_{j, k} .
$$

Now the problem of estimating $f$ based on noisy observation of $K f$ becomes the problem of estimating the wavelet coefficients of $K f$. Suppose $Y(t)$ is observed as in (1). We form the empirical wavelet coefficients $y_{j, k}=\int \psi_{j, k} d Y(t)$ and decompose it as

$$
y_{j, k}=\theta_{j, k}+z_{j, k}
$$

where $\theta_{j, k}=\left\langle K f, \psi_{j, k}\right\rangle$ are the true wavelet coefficients of $K f$ and $z_{j, k}=\int \psi_{j, k} d W(t)$ are the wavelet coefficient of a Brownian motion. Now the noise $z_{j, k}$ are i.i.d. normal since the wavelets $\psi_{j, k}$ are orthonormal.

Abramovich \& Silverman (1998) apply a term-by-term thresholding rule to estimate the wavelet coefficients of $K f$ and map back by $K^{-1}$ to yield the resulting vaguelet-wavelet decomposition estimator $\hat{f}_{V W D}$ :

$$
\hat{f}_{V W D}=\sum_{j, k} \eta_{\lambda}\left(y_{j, k}\right) \beta_{j} w_{j, k} .
$$


With a properly chosen threshold, Abramovich \& Silverman (1998) showed that the estimator is within a logarithmic factor from the minimax risk.

The VWD estimator is numerically stable because wavelet thresholding has been used. The estimate of $K f$ is a linear combination of only a small number of wavelets $\psi_{j, k}$. In cases where the $K^{-1} \psi_{j, k}$ have to be numerically calculated, it is only necessary to find those

$K^{-1} \psi_{j, k}$ that correspond to nonzero coefficients. See Abramovich \& Silverman (1998) for more details.

\section{The Block Thresholding and VWD Approach}

The VWD procedure presented in Abramovich \& Silverman (1998) is conceptually appealing. However, the term-by-term thresholding method used in estimating the wavelet coefficients of $K f$ has drawbacks. The difficulty is mainly caused by the relative inefficiency in estimating the wavelet coefficients individually without using information about other coefficients. The mean squared error of the resulting estimator has a logarithmic penalty.

The estimation accuracy can be improved by using the block thresholding methods. Block thresholding methods threshold the empirical wavelet coefficients in groups rather than individually, making simultaneous decisions to keep or discard all the coefficients within a block. These methods increase estimation precision by utilizing information about neighboring wavelet coefficients.

\subsection{Block Thresholding Method}

In the settings of nonparametric regression and the white noise model, Cai (1999) introduced a block thresholding estimator, BlockJS, based on the block projection oracle inequality. It is shown that the estimator achieves simultaneously three goals: adaptivity, spatial adaptivity, and computational efficiency. The estimator enjoys a higher degree of adaptivity than the standard term-by-term thresholding methods.

Suppose we observe a noisy sampled function $g$ :

$$
y_{i}=g(i / n)+\epsilon z_{i}, \quad i=1,2, \cdots, n\left(=2^{J}\right)
$$

where the $z_{i}$ are i.i.d. and $N(0,1)$. We wish to recover the unknown function $g$ based on the sample. The BlockJS estimator can be described as follows.

1. Transform the data into the wavelet domain via the discrete wavelet transform.

2. At each resolution level $j$, group the empirical wavelet coefficients $\left(\tilde{\theta}_{j}\right.$. $)$ into disjoint blocks $b_{i}^{j}$ of length $L=\log n$. Let $\lambda=4.50524$ and $S_{j i}^{2}=\sum_{(j, k) \in b_{i}^{j}} \tilde{\theta}_{j, k}^{2}$. Within each block $b_{i}^{j}$, estimate the coefficients simultaneously via a shrinkage rule

$$
\hat{\theta}_{j, k}=\left(1-\lambda L \sigma^{2} / S_{j i}^{2}\right)_{+} \tilde{\theta}_{j, k}, \quad \text { for all }(j, k) \in b_{i}^{j}
$$

3. Apply the inverse discrete wavelet transform to the denoised wavelet coefficients to yield the estimate of the function. 
The block length $L=\log n$ is chosen based on the compromise of global and local adaptivity. The threshold $\lambda=4.50524$ is selected according to a block thresholding oracle inequality and a minimax criterion. See Cai (1999) for further details.

The block thresholding approach, together with the vaguelet-wavelet decomposition, can be applied to the linear inverse problems. We will state in detail the estimation procedure in Section 3.2 below. As shown in Section 4 and Section 5.1, the estimator has some very attractive properties.

\subsection{The Estimation Procedure}

A function $g \in L^{2}(\mathbb{R})$ can be expanded in an inhomogeneous orthonormal wavelet basis:

$$
g(t)=\sum_{k}\left\langle g, \phi_{l, k}\right\rangle \phi_{l, k}(t)+\sum_{j \geq l} \sum_{k}\left\langle g, \psi_{j, k}\right\rangle \psi_{j, k}(t) .
$$

The terms $\sum_{k}\left\langle g, \phi_{l, k}\right\rangle \phi_{l, k}$ represent the gross structure of the function, and the terms $\sum_{k}\left\langle g, \psi_{j, k}\right\rangle \psi_{j, k}$ represent finer and finer detail structure of the function $g$ as the resolution level $j$ increases.

Without loss of generality, let us assume that $\phi$ and $\psi$ have the same support with length $N$. In this and later sections, we are interested in estimating functions supported in a fixed finite interval $I \subset \mathbb{R}$. We shall chose the gross-structure index $l$ such that $2^{-l}<|I| /(2 N)$. Since the wavelets $\phi$ and $\psi$ are compactly supported and the interval $I$ is finite, there are only a finite number of coefficients at each resolution level $j$ which may be nonzero for functions supported in $I$. Let

$$
h_{j}=\min \left\{k: \operatorname{supp}\left(\psi_{j, k}\right) \cap I \neq \emptyset\right\} \text {, and } H_{j}=\max \left\{k: \operatorname{supp}\left(\psi_{j, k}\right) \cap I \neq \emptyset\right\} .
$$

It is easy to see that the number of possible nonzero coefficients at level $j$ is $H_{j}-h_{j}+1 \sim$ $2^{j}|I|+2 N$ (see also Donoho (1995)). Then if $g$ is supported in $I$, we have the expansion:

$$
g(t)=\sum_{k=h_{l}}^{H_{l}}\left\langle g, \phi_{l, k}\right\rangle \phi_{l, k}(t)+\sum_{j \geq l} \sum_{k=h_{j}}^{H_{j}}\left\langle g, \psi_{j, k}\right\rangle \psi_{j, k}(t) .
$$

Method: We will assume that we have the white noise observations

$$
d Y(t)=(K f)(t) d t+\frac{\sigma}{\sqrt{n}} d Z(t), \quad t \in \mathbb{R},
$$

where $Z(t)$ is a standard Brownian motion and $K$ is a homogeneous operator of index $\gamma$. We wish to recover $f$, a function known to be supported in a finite interval $I \subset \mathbb{R}$. Our goal is to estimate $f$ with "small" worst case $\operatorname{risk} \sup _{\mathcal{F}} E\|\hat{f}-f\|_{2}^{2}$, where $\mathcal{F}$ is a suitable class of Besov spaces.

We first form the empirical wavelet coefficients of $K f$ :

$$
y_{j, k}=\int \phi_{j, k}(t) d Y(t), \quad h_{j} \leq k \leq H_{j}, j \geq l
$$


and

$$
\tilde{y}_{l, k}=\int \phi_{l, k}(t) d Y(t), \quad h_{l} \leq k \leq H_{l}
$$

Then $y_{j, k}$ can be written as

$$
y_{j, k}=\theta_{j, k}+\sigma n^{-1 / 2} z_{j, k}
$$

with $\theta_{j, k}=\left\langle K f, \psi_{j, k}\right\rangle$ and $z_{j, k} \stackrel{i i d}{\sim} N(0,1)$, and similarly

$$
\tilde{y}_{l, k}=\xi_{l, k}+\sigma n^{-1 / 2} \tilde{z}_{l, k}
$$

with $\xi_{l, k}=\left\langle K f, \phi_{j, k}\right\rangle$ and $\tilde{z}_{l, k}$ iid $N(0,1)$ and independent of $z_{j, k}$ 's.

Let $J=\log _{2} n$. At each resolution level $j \leq J$, we group the empirical wavelet coefficients $\left\{y_{j, k}, h_{j} \leq k \leq H_{j}\right\}$, into nonoverlapping blocks $b_{i}^{j}$ of length $L=\log n$ :

$$
b_{i}^{j}=\left\{(j, k):(i-1) L+h_{j} \leq k \leq i L+h_{j}-1\right\} .
$$

Let $S_{j, i} \equiv \sum_{(j, k) \in b_{i}^{j}} y_{j, k}^{2}$ denote the sum of squared empirical coefficients in block $b_{i}^{j}$. We then apply a James-Stein type shrinkage rule to each block $b_{i}^{j}$,

$$
\hat{\theta}_{j, k}=\left(1-\lambda n^{-1} L \sigma^{2} / S_{j, i}^{2}\right)_{+} y_{j k} \quad \text { for }(j, k) \in b_{i}^{j},
$$

where $\lambda$ is the root of the equation $\lambda-\log \lambda-(3+4 \gamma)=0$ (see Remark 2 below).

The "estimate" of $K f$ is given by

$$
\widehat{K f}(t)=\sum_{k=h_{l}}^{H_{l}} \tilde{y}_{l, k} \phi_{l, k}(t)+\sum_{j \geq l}^{J} \sum_{k=h_{j}}^{H_{j}} \hat{\theta}_{j, k} \psi_{j, k}(t) .
$$

Mapping back by $K^{-1}$ we obtain the estimate of $f$ :

$$
\hat{f}_{n}(t)=\sum_{k=h_{l}}^{H_{l}} \tilde{y}_{l, k}\left(K^{-1} \phi_{l, k}\right)(t)+\sum_{j \geq l}^{J} \sum_{k=h_{j}}^{H_{j}} \hat{\theta}_{j, k} \beta_{j} w_{j k}(t) .
$$

Remark 1: If the number of possible nonzero coefficients at level $j, H_{j}-h_{j}+1$, is not divisible by $L$, then one or both of the blocks at the boundary is shortened to ensure all the blocks are nonoverlapping.

Remark 2: The block length $L=\log n$ is selected based on the compromise of global and local adaptivity. The thresholding constant $\lambda$ is chosen according to the block projection oracle inequality derived in Cai (1999). With the given block length and threshold level, the estimator achieves both global and local adaptivity simultaneously. See Sections 4 and 5.1 for detailed results. The root $\lambda_{*}$ of the equation $\lambda-\log \lambda-\tau=0$ with $\tau>1$ can be written as

$$
\lambda_{*}=\tau+\log (\tau+\log (\tau+\log (\tau+\cdots))) .
$$

Remark 3: The threshold used here is larger than the threshold $\lambda_{*}=4.505 \ldots$ used in Cai (1999) for estimating the regression function. This is similar to the case of term by term threshold used in Abramovich \& Silverman (1998). The universal term by term threshold for estimating $f$ is given as $\lambda=\sqrt{2(1+2 \gamma) \log n}$, which is larger than the universal threshold for estimating $K f$ by a factor of $\sqrt{1+2 \gamma}$. 


\section{Asymptotic Results}

As is traditional in the wavelet literature, we investigate the adaptivity of the estimator (17) over Besov spaces $B_{p, q}^{\alpha}$. Roughly speaking, the Besov function norm $\|f\|_{B_{p, q}^{\alpha}}$ of a function $f \in B_{p, q}^{\alpha}$ quantifies the size in an $L_{p}$ sense of the derivative of $f$ of order $\alpha$, with $q$ giving a finer gradation; for a precise definition of the Besov function norm see DeVore and Popov (1988). We will use an equivalent sequence norm for functions in $B_{p, q}^{\alpha}$.

Suppose $\alpha>0,1 \leq p \leq \infty, 1 \leq q \leq \infty$ and suppose the mother wavelet $\psi$ is $r$-regular with $r>\alpha+\gamma$. Let $\tau_{l, k}=\left\langle f, \phi_{l, k}\right\rangle$ and $d_{j, k}=\left\langle f, \psi_{j, k}\right\rangle$. Then the Besov sequence norm of the wavelet coefficients of a function $f$ is defined by

$$
\left\|\tau_{l} .\right\|_{p}+\left(\sum_{j \geq l}\left(2^{j s}\left\|d_{j} .\right\|_{p}\right)^{q}\right)^{1 / q} .
$$

where $s=\alpha+1 / 2-1 / p$. It is an important fact (Meyer 1992) that the Besov function norm $\|f\|_{B_{p, q}^{\alpha}}$ is equivalent to the sequence norm of the wavelet coefficients of $f$. We define the Besov class $B_{p, q}^{\alpha}(M)$ to be the set of all functions supported on the interval $I$ and whose Besov sequence norm is less than $M$. The special case of $p=q=\infty$ corresponds to the traditional Hölder smoothness class.

Denote the minimax risk over a function class $\mathcal{F}$ by

$$
R(\mathcal{F}, n)=\inf _{\hat{f}_{n}} \sup _{f \in \mathcal{F}} E\left\|\hat{f}_{n}-f\right\|_{2}^{2}
$$

where $\hat{f}_{n}$ are estimators based on the observations (10). Donoho (1995) showed that the minimax risk for estimating $f$ based on (10) over a Besov class $B_{p, q}^{\alpha}(M)$ is given by

$$
R\left(B_{p, q}^{\alpha}(M), n\right) \asymp n^{-2 \alpha /(1+2 \alpha+2 \gamma)}, \quad n \rightarrow \infty
$$

If attention is restricted to linear estimates, the corresponding minimax rate of convergence is $n^{-\rho^{\prime}}$, with

$$
\rho^{\prime}=\frac{2 \alpha+\left(1 / p_{-}-1 / p\right)}{\alpha+\gamma+1 / 2+\left(1 / p_{-}-1 / p\right)}, \quad \text { where } p_{-}=\max (p, 2) .
$$

So the minimax linear rate is strictly slower than the minimax rate when $p<2$.

We will assume the following. The mother wavelet $\psi$ is $r$-regular and the operator $K$ is linear and homogeneous with index $\gamma$. The operator $K^{-1}$ maps a function $g$ supported on an interval to another function $K^{-1} g$ supported on the same interval. Let $w_{j, k}=K^{-1} \psi_{j, k} / 2^{\gamma j}$. There exists some constant $A>0$ such that

$$
\left\|\sum_{j \geq l} \sum_{k} a_{j, k} w_{j, k}(t)\right\|^{2} \leq A\left\|\left(a_{j, k}\right)\right\|_{\ell^{2}}^{2}
$$

We will call these conditions as conditions (C).

The following result shows that the estimator, without knowing the degree or amount of smoothness of the underlying function, attains the exact optimal convergence rate over a range of Besov classes that one could achieve knowing the regularity. 
Theorem 1 Suppose we observe $Y(t)$ as in (10) and suppose the wavelet $\psi$ and the operator $K$ satisfy conditions $(C)$. Let the estimator $\hat{f}_{n}$ be defined as in (15) and (17). Then

$$
\sup _{f \in B_{p, q}^{\alpha}(M)} E\left\|\hat{f}_{n}-f\right\|_{2}^{2} \leq C n^{-\frac{2 \alpha}{1+2 \alpha+2 \gamma}}
$$

for all $0<\alpha<r-\gamma, 0<M<\infty, 2 \leq p \leq \infty$ and $1 \leq q \leq \infty$.

The next theorem addresses the case of $p<2$, and shows that the estimator achieves advantages over linear methods even at the level of rates.

Theorem 2 Suppose the wavelet $\psi$ and the operator $K$ satisfy conditions $(C)$. The estimator is simultaneously within a logarithmic factor of minimax for $p<2$ :

$$
\sup _{f \in B_{p, q}^{\alpha}(M)} E\left\|\hat{f}_{n}-f\right\|^{2} \leq C n^{-\frac{2 \alpha}{1+2 \alpha+2 \gamma}}(\log n)^{(2 / p-1) /(1+2 \alpha+2 \gamma-(4 \gamma / p))}
$$

for all $0<M<\infty, \max \{1 / p,(1 / p-1 / 2)(1+2 \gamma)\}<\alpha<r-\gamma, 1 \leq p<2$, and $1 \leq q \leq \infty$.

In addition to the global estimation properties, the block thresholding estimator enjoys an interesting denoising property. The estimator, with high probability, removes pure noise completely.

Theorem 3 If the target function is the zero function $f \equiv 0$, then, with probability tending to 1 as $n \rightarrow \infty$, the estimator is also the zero function, i.e., there exist universal constants $P_{n}$ such that

$$
P\left(\hat{f}_{n} \equiv 0\right) \geq P_{n} \rightarrow 1, \quad \text { as } n \rightarrow \infty .
$$

The proofs of these theorems are given in Section 7.

\section{Estimating a derivative}

In this section we consider the problem of estimating the derivative of a function $g$. This fits into the inverse problems framework of (10) by setting $K$ to be the integration operator, i.e., $K f(t)=\int_{-\infty}^{t} f(x) d x$, and $g=K f$.

The object of interest is $f$, the derivative of $g=K f$. In this case, the index of the operator $K$ is $\gamma=1$ and the threshold $\lambda=7+\log (7+\log (7+\log (7+\cdots))) \doteq 9.221$.

Now $K^{-1}$ is the differentiation operator, so $K^{-1} g=g^{\prime}$. The vaguelets $\left(w_{j, k}\right)$ are obtained from dilations and translations of the function $\psi^{\prime}$ :

$$
w_{j, k}(t)=2^{j / 2} \psi^{\prime}\left(2^{j} t-k\right) .
$$

Let $\left(v_{j, k}\right)$ be obtained from the function $-\psi^{(-1)}\left(=-\int_{-\infty}^{t} \psi(x) d x\right)$ :

$$
v_{j, k}(t)=-2^{j / 2} \psi^{(-1)}\left(2^{j} t-k\right) .
$$


It is shown in Lee (1997) that, when $\psi$ is $r$-regular with $r>3 / 2,\left(u_{j, k}\right)$ and $\left(v_{j, k}\right)$ are two collections of biorthogonal vaguelets. See also Donoho (1995). Hence $\left(u_{j, k}\right)$ form a Riesz basis and so is "almost" orthonormal. That is, there exist constants $B>b>0$ such that

$$
b\left\|\left(a_{j, k}\right)\right\|_{\ell_{2}} \leq\left\|\sum a_{j, k} u_{j, k}\right\|_{2} \leq B\left\|\left(a_{j, k}\right)\right\|_{\ell_{2}}
$$

for every sequence $\left(a_{j, k}\right)$. It is easy to verify that conditions $(\mathrm{C})$ hold.

In this case, besides the global adaptivity discussed in Section 4, the derivative estimator $\hat{f}_{n}$, given in (15) and (17), also enjoys local adaptivity for estimating the function at a point.

\subsection{Local adaptation}

For functions of spatial inhomogeneity, the local smoothness of the functions varies significantly from point to point. Global risk measures such as (2) cannot wholly reflect the local adaptivity of the estimators. It is more appropriate to use the expected loss at the point for spatial adaptivity,

$$
R\left(\hat{f}\left(t_{0}\right), f\left(t_{0}\right)\right)=E\left(\hat{f}\left(t_{0}\right)-f\left(t_{0}\right)\right)^{2} .
$$

We measure the local smoothness of a function at a point by its local Hölder smoothness index. Let us define the local Hölder class $\Lambda^{\alpha}\left(M, t_{0}, \delta\right)$ as follows.

$$
\Lambda^{\alpha}\left(M, t_{0}, \delta\right)=\left\{f:\left|f^{(\lfloor\rfloor\rfloor)}(t)-f^{(\lfloor\alpha\rfloor)}\left(t_{0}\right)\right| \leq M\left|t-t_{0}\right|^{\alpha^{\prime}} \quad t \in\left(t_{0}-\delta, t_{0}+\delta\right)\right\}
$$

where $\lfloor\alpha\rfloor$ is the largest integer less than $\alpha$ and $\alpha^{\prime}=\alpha-\lfloor\alpha\rfloor$.

It is well known that for global estimation, it is possible to achieve complete adaptation for free in terms of the convergence rate across a range of function classes. For instance, as shown in Theorem 1, the estimator attains the optimal rate of convergence simultaneously over a range of function classes. For estimation at a point, however, one must pay a price for not knowing the smoothness of the underlying function.

Lepski (1990) and Brown and Low (1996) show that, in the case of estimating a drift function (i.e. $g=K f$ in (10)) at a point, it is impossible to achieve adaption to unknown smoothness without loss of efficiency, even when the function is known to belong to one of two Hölder classes. Therefore, local adaptation can not be achieved "for free". The minimum loss of efficiency is a logarithmic factor for estimating a function of unknown degree of local Hölder smoothness at a point. See Lepski (1990) and Brown and Low (1996). See also Donoho and Johnstone (1995).

A similar result holds for estimating a derivative at a point. Denote the minimax risk for estimating functions at a point $t_{0}$ over a function class $\mathcal{F}$ by

$$
R_{n}\left(\mathcal{F}, t_{0}\right)=\inf _{\hat{f}} \sup _{\mathcal{F}} E\left(\hat{f}\left(t_{0}\right)-f\left(t_{0}\right)\right)^{2}
$$

The minimax rate of convergence for estimating $f\left(t_{0}\right)$ based on (10) with $\alpha$ known is $n^{-\rho}$ where $\rho=2 \alpha /(3+2 \alpha)$. One may use the proof in Brown and Low (1996) with only minor changes to show that the risk for adaptively estimating $f$ at a point based on (10) is at least of order $\left(n^{-1} \log n\right)^{2 \alpha /(3+2 \alpha)}$ for $f \in \Lambda^{\alpha}\left(M, t_{0}, \delta\right)$ with $\alpha$ unknown. We call $\left(n^{-1} \log n\right)^{2 \alpha /(3+2 \alpha)}$ the local adaptive minimax rate for estimating $f$ at a point.

The following theorem shows that the estimator given in (17) achieves the local adaptive minimax rate over a range of local Hölder classes. 
Theorem 4 Suppose the wavelet $\psi$ is $r$-regular with $r>3 / 2$ and $r \geq \alpha+1$. Let $t_{0}$ be a fixed interior point of $I$. Then the estimator $\hat{f}_{n}$ given in (15) and (17) satisfies

$$
\sup _{f \in \Lambda^{\alpha}\left(M, t_{0}, \delta\right)} E\left\{\hat{f}_{n}\left(t_{0}\right)-f\left(t_{0}\right)\right\}^{2} \leq C\left(n^{-1} \log n\right)^{2 \alpha /(3+2 \alpha)} .
$$

Remark 3: In general, if a linear operator $K$ satisfies conditions (C), then it can be shown that the estimator $\hat{f}_{n}$ satisfies

$$
\left.\sup _{f \in \Lambda^{\alpha}\left(M, t_{0}, \delta\right)} E\left\{\hat{f}_{n}^{(} t_{0}\right)-f\left(t_{0}\right)\right\}^{2} \leq C\left(n^{-1} \log n\right)^{2 \alpha /(1+2 \alpha+2 \gamma)} .
$$

Remark 4: The choice of $L=\log n$ is important for achieving the optimal local adaptivity. The result does not hold if $L=(\log n)^{1+\delta}, \delta>0$.

\subsection{Discrete Data}

In practice one observes discrete data instead of a continuous-time white noise process (10). Similar to wavelets, a system of vaguelets has a corresponding Discrete Vaguelet Transform (DVT). The transform is no longer orthogonal and the corresponding DVT varies for different operators $K$ in inverse problems. Kolaczyk (1996) provides efficient algorithms for the DVT and its inverse for the Radon transform, each requiring $O(n \log n)$ operations. In the general case, performing the DVT and its inverse may be computationally expensive.

In this section, we discuss the numerical implementation of the block thresholding derivative estimator when sampled data are observed. Suppose that $f$ is a function of interest and we observe noisy data

$$
y_{i}=\int_{0}^{i / n} f(t) d t+\sigma z_{i}, \quad i=1, \ldots, n,
$$

where $n=2^{J}$ for some positive integer $J$ and $z_{i} \stackrel{i i d}{\sim} N(0,1)$. Again, denote $g(t)=$ $\int_{0}^{t} f(x) d x$, so $f=g^{\prime}$. To avoid complications caused by boundary effects, we assume here that $f(0)=f(1)$ and $g(0)=g(1)$. We shall use the periodic discrete wavelet transform and coiflets with regularity exceeding $3 / 2$.

We begin with an approximation problem where no noise is present. Suppose a sampled function $g_{s, n}=(g(1 / n), g(2 / n), \cdots, g(n / n))$, where $n=2^{J}$, is given. We wish to have a fast wavelet algorithm to approximate $f_{s, n}=(f(1 / n), f(2 / n), \cdots, f(n / n))$. Our numerical algorithm is based on the following approximation results.

Theorem 5 Suppose the wavelets $\{\phi, \psi\}$ are a pair of $r$-regular coiflets. Let

$$
f_{n}(t)=\sum_{k=1}^{n} \sum_{i=1}^{n} n^{-1 / 2} g(i / n)\left\langle\phi_{J i},-\left(\phi_{J k}\right)^{\prime}\right\rangle \phi_{J k}(t)
$$

and let $\tilde{f}_{s, n}=D \cdot g_{s, n}$ where $D$ is a $n \times n$ matrix with entries $D_{k, i}=\left\langle\phi_{J i},-\left(\phi_{J k}\right)^{\prime}\right\rangle$. Then

$$
\begin{aligned}
\sup _{f \in \Lambda^{\alpha}(M)}\left\|f_{n}-f\right\|_{2}^{2} & \leq C n^{-2 \alpha} \\
\sup _{f \in \Lambda^{\alpha}(M)}\left\|\tilde{f}_{s, n}-f_{s, n}\right\|_{\infty} & \leq C n^{-\alpha}
\end{aligned}
$$

for all $0<\alpha \leq r$ and $M>0$. 
Interestingly, the values of the approximation $\tilde{f}_{s, n}$ can be computed in $O(n)$ operations via a fast algorithm. We first note that

$$
D_{k i}=\left\langle\phi_{J i},-\left(\phi_{J k}\right)^{\prime}\right\rangle=-2^{J} \int \phi^{\prime}(t) \phi(t-(i-k)) d t .
$$

Denote $c_{m}=\int \phi^{\prime}(t) \phi(t-m) d t$, so $D_{k, i}=-2^{J} c_{i-k}$. Suppose $\phi$ is supported on $[0, B+1]$ and satisfies the dilation equation

$$
\phi(t)=\sum_{i=0}^{B+1} h_{i} \sqrt{2} \phi(2 t-i) .
$$

It follows that $c_{m}$, which is non-vanishing only if $|m| \leq B$, satisfies the equation

$$
c_{m}=2 \sum_{i, j=0}^{B+1} h_{i} h_{j} c_{2 m+i-j}=2 \sum_{k=-B}^{B}\left(\sum_{j=0}^{B+1} h_{j} h_{k-2 m+j}\right) c_{k} .
$$

The $c_{m}$ are thus the eigenvector with eigenvalue $1 / 2$ of the matrix $H$ with entries

$$
H_{m, k}=\sum_{j=0}^{B+1} h_{j} h_{k-2 m+j}
$$

for $|m|,|k| \leq B$. If $\psi$ has two vanishing moments, then the matrix $H$ does have the eigenvalue $1 / 2$ and it is nondegenerate (Daubechies (1994)). Moreover, Beylkin (1992) proves that

$$
\sum m c_{m}=-1
$$

This fixes the normalization of the $c_{m}$, so that they are uniquely determined. The values of the $c_{m}$ need only be computed once directly from the $h_{k}$ and stored in a look-up table. The values of $\tilde{f}_{s, n}$ can then be computed by a sequence of finite length filtering on $g_{s, n}$ which requires $O(n)$ operations.

Now we are ready to state the numerical algorithm implementing the block thresholding estimator. The algorithm consists of four steps and the total complexity is $O(n)$.

1. Transform the data $y$ given in (26) into wavelet domain via the discrete wavelet transform.

2. At each resolution level $j$, group the empirical wavelet coefficients into disjoint blocks $b_{i}^{j}$ of length $L=\log n$. Let $\lambda_{*}=9.221$. Within each block $b_{i}^{j}$, estimate the coefficients simultaneously via a shrinkage rule

$$
\hat{\theta}_{j, k}=\left(1-\lambda_{*} L \sigma^{2} / S_{j, i}^{2}\right)_{+} y_{j k}
$$

3. Apply the inverse discrete wavelet transform to the denoised wavelet coefficients to get the "estimate" $\hat{g}(i / n)$ of $g(i / n)=\int_{0}^{i / n} f(x) d x$.

4. Obtain the estimate of $f$ at the sample points by a sequence of finite length filtering on $\hat{g}(i / n)$ with the filter coefficients $\left(-n c_{m}\right)$ :

$$
\hat{f}(k / n)=-n \sum_{i} c_{i-k} \hat{g}(i / n) .
$$




\section{Concluding Remarks}

Block thresholding serves as a bridge between the traditional shrinkage estimators in normal decision theory and the more recent wavelet function estimation. This connection enables us to develop a class of near-optimal wavelet estimators all of which may be useful in different estimation situations. We have focused on the James-Stein shrinkage rule in the present paper. Other shrinkage rules can be used as well. For example, a "hard" thresholding rule can be used for estimating $\theta_{j, k}$ within a block $b_{i}^{j}$ :

$$
\hat{\theta}_{j, k}=y_{j, k} \cdot I\left(S_{j, i}^{2}>\lambda n^{-1} L \sigma^{2}\right), \text { for }(j, k) \in b_{i}^{j} .
$$

Other blocking rules can also be used. For example, the method of Cai \& Silverman (1999) can be modified for the use in linear inverse problems.

The block thresholding estimator can also be modified by averaging over different block centers. In the case of nonparametric regression, the averaged estimator often has superior numerical performance, at the cost of higher computational complexity. See Cai (1999) and Hall, et al. (1997).

\section{Proofs}

Assume that the mother wavelet $\psi$ and the operator $K$ satisfy conditions $(\mathrm{C})$. Then the function $f$ can be written as

$$
f(t)=\sum_{k=h_{l}}^{H_{l}} \xi_{l, k}\left(K^{-1} \phi_{l, k}\right)(t)+\sum_{j=l}^{\infty} \sum_{k=h_{j}}^{H_{j}} \theta_{j, k} 2^{\gamma j} w_{j, k}(t)
$$

where $\xi_{l, k}=\left\langle K f, \phi_{l, k}\right\rangle, w_{j, k}$ are the vaguelets and $\theta_{j, k}=\left\langle K f, \psi_{j, k}\right\rangle$ are the wavelet coefficients of $K f$. Under the assumptions of Theorem 1, the function $f$ is supported on the interval $I$ and is in Besov class $B_{p, q}^{\alpha}(M)$. So,

$$
\left(\sum_{j=l}^{\infty}\left(2^{j s}\left(\sum_{k=h_{j}}^{H_{j}}\left|d_{j, k}\right|^{p}\right)^{1 / p}\right)^{q}\right)^{1 / q} \leq M
$$

where $d_{j, k}=\left\langle f, \psi_{j, k}\right\rangle$ and $s=\alpha+1 / 2-1 / p$. As noted in Abramovich \& Silverman (1998, pp. 128), the operator $K$ maps a Besov space $B_{p, q}^{\alpha}$ to another Besov space $B_{p, q}^{\alpha+\gamma}$ and there exists a constant $M_{1}>0$ such that

$$
\left(\sum_{j=l}^{\infty}\left(2^{j s^{\prime}}\left(\sum_{k=h_{j}}^{H_{j}}\left|\theta_{j, k}\right|^{p}\right)^{1 / p}\right)^{q}\right)^{1 / q} \leq M_{1}
$$

for every function $f$ satisfying (33) where $\theta_{j, k}=\left\langle K f, \psi_{j, k}\right\rangle$ and $s^{\prime}=s+\gamma$. 


\subsection{Proof of Theorem $1 \& 2$}

We first state a result which follows directly from the block projection oracle inequality and Lemma 2 in Cai (1999).

Lemma 1 Let $x_{i}=\mu_{i}+\epsilon z_{i}, \quad i=1, \cdots, L(=\log n)$, and let $\hat{\mu}_{i}=\left(1-\lambda L \epsilon^{2} / S^{2}\right)_{+} x_{i}$. where $S^{2}=\|x\|^{2}$ and $\lambda$ is the root of the equation $\lambda-\log \lambda-(3+4 \gamma)=0$. Then

$$
E\|\hat{\theta}-\theta\|_{2}^{2} \leq \lambda\left(\|\mu\|^{2} \wedge L \epsilon^{2}\right)+2 \epsilon^{2} n^{-(1+2 \gamma)} .
$$

The following elementary inequalities concerning different norms are also needed.

Lemma 2 Let $x \in \mathbb{R}^{m}$, and $0<p_{1} \leq p_{2} \leq \infty$. Then the following inequalities hold:

$$
\|x\|_{p_{2}} \leq\|x\|_{p_{1}} \leq m^{\frac{1}{p_{1}}-\frac{1}{p_{2}}}\|x\|_{p_{2}} .
$$

Let $y_{j, k}, \hat{\theta}_{j, k}$ and $\hat{f}_{n}$ be given as in (13), (15) and (17), respectively. It follows from the triangle inequality and the fact that $w_{j, k}$ are vaguelets that

$$
\begin{aligned}
E\left\|\hat{f}_{n}-f\right\|_{2}^{2} \leq & 2 E\left\|\sum_{k=h_{l}}^{H_{l}}\left(\tilde{y}_{j, k}-\xi_{j, k}\right)\left(K^{-1} \phi_{l, k}\right)(t)\right\|_{2}^{2}+2 A \sum_{j=l}^{J} \sum_{k=h_{j}}^{H_{j}} 2^{2 \gamma j}\left(\hat{\theta}_{j, k}-\theta_{j, k}\right)^{2} \\
& +2 A \sum_{j=J+1}^{\infty} \sum_{k=h_{j}}^{H_{j}} 2^{2 \gamma j} \theta_{j, k}^{2} \\
\equiv & T_{1}+T_{2}+T_{3} .
\end{aligned}
$$

Since $E\left(\tilde{y}_{j, k}-\xi_{j, k}\right)^{2}=\sigma^{2} n^{-1}$ and $H_{l}-h_{l}+1$ is fixed and finite, it follows from the triangle inequality that $T_{1}=O\left(n^{-1}\right)$. We now bound the other two terms and divide into two cases: $p \geq 2$ and $p<2$.

The case $p \geq 2$ : It follows from the Besov norm constraint (34) that $2^{j s^{\prime}}\left(\sum_{k=h_{j}}^{H_{j}}\left|\theta_{j k}\right|^{p}\right)^{1 / p} \leq$ $M_{1}$. Lemma 2 yields that for $p \geq 2, \sum_{k=h_{j}}^{H_{j}}\left|\theta_{j k}\right|^{2} \leq M_{1}^{2} 2^{-j 2(\alpha+\gamma)}$. Denote by $C$ a generic constant that may vary from place to place. Then

$$
T_{3}=\sum_{j=J+1}^{\infty} 2^{\gamma j} \sum_{k=h_{j}}^{H_{j}} \theta_{j k}^{2} \leq \sum_{j=J+1}^{\infty} M_{1}^{2} 2^{-j 2 \alpha} \leq C n^{-2 \alpha}=o\left(n^{-2 \alpha /(1+2 \alpha+2 \gamma)}\right) .
$$

Now consider the term $T_{2}$. Denote by $Q_{j, i}^{2}=\sum_{k \in b_{i}^{j}} \theta_{j, k}^{2}$ the sum of squared coefficients within the block $b_{i}^{j}$ and let $J_{1}$ be an integer satisfying $2^{J_{1}} \asymp n^{1 /(1+2 \alpha+2 \gamma)}$. With $\epsilon=n^{-1 / 2} \sigma$, Lemma 1 together with the fact that $H_{j}-h_{j}+1 \sim 2^{j}|I|+2 N$ yield

$$
\begin{aligned}
T_{2} & =\sum_{j=l}^{J} \sum_{k=h_{j}}^{H_{j}} 2^{2 \gamma j} E\left(\hat{\theta}_{j k}-\theta_{j k}\right)^{2} \leq \lambda \sum_{j=l}^{J} 2^{2 \gamma j} \sum_{i}\left(Q_{j, i}^{2} \wedge L n^{-1} \sigma^{2}\right)+C n^{-1} \sigma^{2} \\
& \leq \lambda \sum_{j=l}^{J_{1}-1} 2^{2 \gamma j} \sum_{i} L n^{-1} \sigma^{2}+\lambda \sum_{j=J_{1}}^{J} \sum_{i} Q_{j, i}^{2}+C n^{-1} \sigma^{2} \leq C n^{-2 \alpha /(1+2 \alpha+2 \gamma)} .
\end{aligned}
$$


Putting together the three terms $T_{1}, T_{2}$ and $T_{3}$, we have

$$
\sup _{f \in B_{p, q}^{\alpha}(M)} E\left\|\hat{f}_{n}-f\right\|_{2}^{2} \leq C n^{-2 \alpha /(1+2 \alpha+2 \gamma)}, \quad \text { for } p \geq 2 .
$$

The case $p<2$ : Since $\theta$ satisfies the condition (34), Lemma 2 yields $\sum_{k=h_{j}}^{H_{j}}\left|\theta_{j k}\right|^{2} \leq$ $M_{1}^{2} 2^{-2 s^{\prime} j}$. The assumption $\alpha \geq 1 / p$ implies that $T_{3}$ is of higher order:

$$
T_{3}=\sum_{j=J+1}^{\infty} 2^{2 \gamma j} \sum_{k=h_{j}}^{H_{j}} \theta_{j k}^{2} \leq \sum_{j=J+1}^{\infty} M_{1}^{2} 2^{-2 s^{\prime} j+2 \gamma j} \leq C n^{-2 \alpha-1+2 / p}=o\left(n^{-2 \alpha /(1+2 \alpha+2 \gamma)}\right) .
$$

Now consider the term $T_{2}$. We state the following lemma.

Lemma 3 Let $0<p<1$ and $S=\left\{x \in \mathbb{R}^{k}: \sum_{i=1}^{k} x_{i}^{p} \leq B, x_{i} \geq 0, i=1, \cdots, k\right\}$. Then for $A>0$,

$$
\sup _{x \in S} \sum_{i=1}^{k}\left(x_{i} \wedge A\right) \leq B \cdot A^{1-p} \text {. }
$$

The proof of Lemma 3 is straightforward since

$$
\sum_{i=1}^{k}\left(x_{i} \wedge A\right)=A \sum_{i=1}^{k}\left(\left(x_{i} / A\right) \wedge 1\right) \leq A \sum_{i=1}^{k}\left(\left(x_{i} / A\right)^{p} \wedge 1\right) \leq B A^{1-p} .
$$

Back to the case $p<2$. Again denote $Q_{j, i}^{2}=\sum_{k \in b_{i}^{j}} \theta_{j k}^{2}$. Lemma (1) yields

$$
T_{2}=\sum_{j=l}^{J} 2^{2 \gamma j} \sum_{k=h_{j}}^{H_{j}} E\left(\hat{\theta}_{j k}-\theta_{j k}\right)^{2} \leq \lambda \sum_{j=l}^{J} 2^{2 \gamma j} \sum_{i}\left(Q_{j, i}^{2} \wedge L n^{-1} \sigma^{2}\right)+C n^{-1} \sigma^{2} .
$$

Let $J_{2}$ be an integer satisfying $2^{J_{2}} \asymp n^{1 /(1+2 \alpha+2 \gamma)}(\log n)^{(2 / p-1) /(1+2 \alpha+2 \gamma-(4 \gamma / p))}$. Then

$$
\begin{aligned}
\lambda \sum_{j=l}^{J_{2}-1} 2^{2 \gamma j} \sum_{i}\left(Q_{j, i}^{2} \wedge L n^{-1} \sigma^{2}\right) & \leq \lambda \sum_{j=l}^{J} 2^{2 \gamma j} \sum_{i} L n^{-1} \sigma^{2} \\
& \leq C n^{-2 \alpha /(1+2 \alpha+2 \gamma)}(\log n)^{(2 / p-1) /(1+2 \alpha+2 \gamma-(4 \gamma / p))} .
\end{aligned}
$$

Note that $\sum_{i}\left(Q_{j, i}^{2}\right)^{p / 2} \leq \sum_{k}\left(\theta_{j, k}^{2}\right)^{p / 2} \leq M_{1} 2^{-j s^{\prime} p}$. Lemma 3 yields

$$
\lambda \sum_{j=J_{2}}^{J} 2^{2 \gamma j} \sum_{i}\left(Q_{j, i}^{2} \wedge L n^{-1} \sigma^{2}\right) \leq C n^{-2 \alpha /(1+2 \alpha+2 \gamma)}(\log n)^{(2 / p-1) /(1+2 \alpha+2 \gamma-(4 \gamma / p))} .
$$

We finish the proof for $p<2$ by combining the three terms,

$$
\sup _{f \in B_{p, q}^{\alpha}(M)} E\left\|\hat{f}_{n}-f\right\|_{2}^{2} \leq C n^{-2 \alpha /(1+2 \alpha+2 \gamma)}(\log n)^{(2 / p-1) /(1+2 \alpha+2 \gamma-(4 \gamma / p))} .
$$




\subsection{Proof of Theorem 3}

The function $K f$ is estimated by zero if and only if all the coefficients are estimated by zero. When $\theta_{j k} \equiv 0$, then from (13) and (15) the probability that a block is estimated by zero is $P\left(\sum_{k \in b_{i}^{j}} z_{j k}^{2} \leq \lambda L\right)$. The total number of blocks is $C n / L$ for some fixed constant $C>0$. Therefore, the probability of $\hat{f}_{n} \equiv 0$ is

$$
\begin{aligned}
P\left(\hat{f}^{*} \equiv 0\right) & =P(\widehat{K f} \equiv 0)=\left[P\left(\sum_{k \in b_{i}^{j}} z_{j k}^{2} \leq \lambda L\right)\right]^{C n / L}=\left[1-P\left(\sum_{k \in b_{i}^{j}} z_{j k}^{2}>\lambda L\right)\right]^{n / L} \\
& \geq\left[\left(1-\frac{1}{n^{1+2 \gamma}}\right)^{n}\right]^{C / L}
\end{aligned}
$$

The last inequality follows from Lemma 2 in Cai (1999) on the tail probability of a chisquare distribution. Let $P_{n}=\left[\left(1-\frac{1}{n^{1+2 \gamma}}\right)^{n}\right]^{C / L}$. Since $\left(1-\frac{1}{n^{1+2 \gamma}}\right)^{n}$ tends to 1 when $\gamma>0$ and to $e^{-1}$ when $\gamma=0$, and $C / L \rightarrow 0$, so $P_{n} \rightarrow 1$ as $n \rightarrow \infty$.

\subsection{Proof of Theorem 4}

For simplicity, we give the proof for Hölder classes $\Lambda^{\alpha}(M)$ instead of local Hölder classes $\Lambda^{\alpha}\left(M, t_{0}, \delta\right)$. For Hölder classes $\Lambda^{\alpha}(M)$ there exists a constant $M_{2}>0$ such that

$$
\left|d_{j, k}\right|=\left|\left\langle f, \psi_{j, k}\right\rangle\right| \leq C 2^{-j(1 / 2+\alpha)} \text {, all } f \in \Lambda^{\alpha}(M) \text {. }
$$

We also note that when $K$ is the integration operator, $\gamma=1$ and $f \in \Lambda^{\alpha}(M)$ implies $K f \in \Lambda^{1+\alpha}(M)$. So,

$$
\left|\theta_{j, k}\right|=\left|\left\langle K f, \psi_{j, k}\right\rangle\right| \leq C 2^{-j(3 / 2+\alpha)}, \text { all } f \in \Lambda^{\alpha}(M) .
$$

The proof of the theorem makes use of the following elementary inequality.

Lemma 4 Let $X_{i}$ be random variables, $i=1, \cdots, n$. Then

$$
E\left(\sum_{i=1}^{n} X_{i}\right)^{2} \leq\left(\sum_{i=1}^{n}\left(E X_{i}^{2}\right)^{1 / 2}\right)^{2} .
$$

Now apply the inequality (45), we have

$$
\begin{aligned}
& E\left(\hat{f}_{n}\left(t_{0}\right)-f\left(t_{0}\right)\right)^{2}=E\left[\sum_{k=h_{l}}^{H_{l}}\left(\tilde{y}_{l, k}-\xi_{l, k}\right)\left(K^{-1} \phi_{l, k}\right)\left(t_{0}\right)+\sum_{j=l}^{J} \sum_{k=h_{j}}^{H_{j}} 2^{j}\left(\hat{\theta}_{j k}-\theta_{j k}\right) w_{j k}\left(t_{0}\right)\right. \\
& \left.\quad+\sum_{j=J+1}^{\infty} \sum_{k=h_{j}}^{H_{j}} 2^{j} \theta_{j k} w_{j k}\left(t_{0}\right)\right]^{2} \\
& \leq\left[\sum_{k}\left|\left(K^{-1} \phi_{l, k}\right)\left(t_{0}\right)\right|\left(E\left(\tilde{y}_{l, k}-\xi_{l, k}\right)^{2}\right)^{1 / 2}+\sum_{j=l}^{J} \sum_{k} 2^{j}\left|w_{j k}\left(t_{0}\right)\right|\left(E\left(\hat{\theta}_{j k}-\theta_{j k}\right)^{2}\right)^{1 / 2}\right. \\
& \left.\quad+\sum_{j=J+1}^{\infty} \sum_{k} 2^{j}\left|\theta_{j k} w_{j k}\left(t_{0}\right)\right|\right]^{2} \\
& \equiv\left(Q_{1}+Q_{2}+Q_{3}\right)^{2} .
\end{aligned}
$$


Since the vaguelets are of compact support, so there are at most $N$ vaguelets $w_{j k}$ at each resolution level $j$ that are nonvanishing at $t_{0}$, where $N$ is the length of the support of the vaguelet $w=\psi^{\prime}$. Denote $K\left(j ; t_{0}\right)=\left\{k: w_{j, k}\left(t_{0}\right) \neq 0\right\}$. Then $\left|K\left(j ; t_{0}\right)\right| \leq N$. It is easy to see that both $Q_{1}$ and $Q_{3}$ are small:

$$
\begin{aligned}
Q_{1} & =\sum_{k}\left|\left(K^{-1} \phi_{l, k}\right)\left(t_{0}\right)\right|\left(E\left(\tilde{y}_{l, k}-\xi_{l, k}\right)^{2}\right)^{1 / 2}=O\left(n^{-1}\right), \\
Q_{3} & =\sum_{j=J+1}^{\infty} \sum_{k \in K\left(j ; t_{0}\right)}\left|\theta_{j k}\right|\left|w_{j k}\left(t_{0}\right)\right| \leq \sum_{j=J}^{\infty} 2^{j} N\left\|\psi^{\prime}\right\|_{\infty} 2^{j / 2} C 2^{-j(3 / 2+\alpha)} \leq C n^{-\alpha} .
\end{aligned}
$$

We now consider the second term $Q_{2}$. Applying Lemma 1 and using (44), we have

$$
\begin{aligned}
Q_{2} & \leq \sum_{j=l}^{J} \sum_{k \in K\left(j ; t_{0}\right)} 2^{j} 2^{j / 2}\left\|\psi^{\prime}\right\|_{\infty}\left(E\left(\hat{\theta}_{j k}-\theta_{j k}\right)^{2}\right)^{1 / 2} \\
& \leq C \sum_{j=l}^{J} 2^{3 j / 2}\left[\left(2^{-j(3+2 \alpha)} \wedge L n^{-1} \epsilon^{2}\right)+L n^{-4} \sigma^{2}\right]^{1 / 2} \leq C\left(n^{-1} \log n\right)^{\alpha /(3+2 \alpha)} .
\end{aligned}
$$

Combining (46), (47) and (48), we have $E\left(\hat{f}_{n}\left(t_{0}\right)-f\left(t_{0}\right)\right)^{2} \leq C\left(n^{-1} \log n\right)^{2 \alpha /(3+2 \alpha)}$.

\subsection{Proof of Theorem 5}

Throughout the proof we assume that the wavelets $\{\phi, \psi\}$ are a pair of $r$-regular coiflets, $n=2^{J}, f \in \Lambda^{\alpha}(M)$ with $f(0)=f(1)$ and $0<\alpha \leq r-1$, and $g(t)=(K f)(t)=\int_{0}^{t} f(x) d x$ with $g(0)=g(1)$. We first state the following lemma which is a consequence of the vanishing moments conditions on the wavelets $\{\phi, \psi\}$.

Lemma 5 Let $h \in \Lambda^{\omega}(M)$ with $0<\omega \leq r$. Then there exists a constant $A>0$, independent of $h$, such that

$$
\left|n^{-1 / 2} h(k / n)-\left\langle h, \phi_{J k}\right\rangle\right| \leq A \cdot n^{-(1 / 2+\omega)} \quad \text { and } \quad\left|\left\langle h, \psi_{j k}\right\rangle\right| \leq A \cdot 2^{-j(1 / 2+\omega)} .
$$

Consequently, if we let $h_{n}(t)=\sum_{i=1}^{n} n^{-1 / 2} h(i / n) \phi_{J, i}(t)$, then

$$
\sup _{h \in \Lambda^{\omega}(M)}\left\|h_{n}-h\right\|_{\infty} \leq C n^{-\omega} \text { and } \sup _{h \in \Lambda^{\omega}(M)}\left\|h_{n}-h\right\|_{2}^{2} \leq C n^{-2 \omega}
$$

for all $0<\omega \leq r$ and $M>0$.

Since $\phi_{J k}$ is compactly supported in $[0,1]$, using integration by parts and the fact that $g(0)=g(1)$, one has

$$
\tau_{J k} \equiv\left\langle f, \phi_{J k}\right\rangle=\left\langle g,-\left(\phi_{J k}\right)^{\prime}\right\rangle .
$$

Let $g_{n}(t)=\sum_{i=1}^{n} n^{-1 / 2} g(i / n) \phi_{J, i}(t)$. The assumption $f \in \Lambda^{\alpha}(M)$ implies $g \in \Lambda^{1+\alpha}(M)$. Lemma 5 yields

$$
\left|\tau_{J k}-\left\langle g_{n},-\left(\phi_{J k}\right)^{\prime}\right\rangle\right| \leq\left\langle\left|g_{n}-g\right|,\left|\left(\phi_{J k}\right)^{\prime}\right|\right\rangle \leq C n^{-(1 / 2+\alpha)}
$$


Rewrite $f_{n}$ in (27) as $f_{n}=\sum_{k}\left\langle g_{n},-\left(\phi_{J k}\right)^{\prime}\right\rangle \phi_{j, k}$, then (28) follows from (49) and (51). Some algebra and (49) and (51) also yield

$$
\sup _{f \in \Lambda^{\alpha}(M)}\left\|f_{n}-f\right\|_{\infty} \leq C n^{-\alpha} .
$$

The approximation of $f$ at the sample point $k / n$ is given by

$$
\tilde{f}_{s, n}(k)=\sum_{i=1}^{n} g(i / n)\left\langle\phi_{J i},-\left(\phi_{J k}\right)^{\prime}\right\rangle
$$

Noting $\tilde{f}_{s, n}(k)=n^{1 / 2}\left\langle f_{n}, \phi_{J k}\right\rangle$, the approximation error is bounded as follows.

$$
\left|\tilde{f}_{s, n}(k)-f(k / n)\right| \leq n^{1 / 2}\left(\left|\tau_{J k}-n^{-1 / 2} f(k / n)\right|+\left|\left\langle f_{n}-f, \phi_{J k}\right\rangle\right|\right) \leq C n^{-\alpha} .
$$

The last inequality follows from (49) and (52). Now (54) yields (29).

\section{Acknowledgment}

It is a pleasure to acknowledge helpful comments by William Studden which improved the presentation of the paper.

\section{References}

[1] Abramovich, F. \& Silverman, B.W. (1998). Wavelet decomposition approaches to statistical inverse problems. Biometrika 85, 115-129.

[2] Beylkin, G. (1992). On the representation of operators in bases of compactly supported wavelets. SIAM J. Num. Anal. 29, 1716-1740.

[3] Brown, L.D. \& Low, M.G. (1996). A constrained risk inequality with applications to nonparametric functional estimation. Ann. Statist. 242524 - 2535.

[4] Cai, T. (1999). Adaptive wavelet estimation: A block thresholding and oracle inequality approach. Ann. Statist. in press.

[5] Cai, T. \& Silverman, B.W. (1999). Incorporating information on neighboring coefficients into wavelet estimation. Technical Report \#98-13, Department of Statistics, Purdue University. Revised March, 1999.

[6] Cohen, A., Daubechies, I., Jawerth, B. \& Vial, P. (1993). Multiresolution analysis, wavelets, and fast algorithms on an interval. Comptes Rendus Acad. Sci. Paris (A). 316, 417-421.

[7] Daubechies, I. (1992). Ten Lectures on Wavelets SIAM: Philadelphia.

[8] Daubechies, I. (1994). Two recent results on wavelets: wavelet bases for the interval, and biorthogonal wavelets diagonalizing the derivative operator. In Schumaker L.L. \& Webb G. (eds), Recent Advances in Wavelet Analysis. Academic Press, 237 - 258. 
[9] Donoho, D.L. (1995). Nonlinear solution of linear inverse problems by waveletvaguelette decomposition. Appl. Comput. Harm. Anal. 2, 101-126.

[10] Donoho, D.L. \& Johnstone, I.M. (1992). Ideal spatial adaptation via wavelet shrinkage. Biometrika, 81, 425-455.

[11] Donoho, D.L. \& Johnstone, I.M. (1995). Neo-classic minimax problems, thresholding, and adaptation. Bernoulli, 2, $39-62$.

[12] Donoho, D.L., Johnstone, I.M., Kerkyacharian, G. \& Picard, D. (1995). Wavelet shrinkage: asymptopia?, J. Roy. Stat. Soc. Ser. B, 57, 301-369.

[13] Gasser, T., Müller, H.-G., Köhler, W., Molinari, L. \& Prader, A. (1984). Nonparametric regression analysis of growth curves. Ann. Statist. $12210-229$.

[14] Hall, P., Kerkyacharian, G. \& Picard, D. (1999). On the minimax optimality of block thresholded wavelet estimators. Statist. Sinica, 9, 33-50.

[15] Hall, P., Penev, S., Kerkyacharian, G. \& Picard, D. (1997). Numerical performance of block thresholded wavelet estimators. Statist. Comput. 7 115-124.

[16] Johnstone, I.M. (1999). Wavelet shrinkage for correlated data and inverse problems: adaptivity results. Statist. Sinica, $\mathbf{9}, 51-84$.

[17] Johnstone, I.M. \& Silverman, B.W. (1990). Speed of estimation in positron emission tomography and related inverse problems. Ann. Statist. 18, 251-280.

[18] Kolaczyk, E. D. (1996). A wavelet shrinkage approach to tomographic image reconstruction. J. Am. Statist. Assoc. 91, 1079-90.

[19] Lee, N. (1997). Wavelet-vaguelet decompositions and homogeneous equations. Ph.D dissertation, Purdue University.

[20] Lepski, O.V. (1990). On a problem of adaptive estimation on white Gaussian noise. Theory of Probability and Appl. 35, 3, 454-466.

[21] Meyer, Y. (1992). Wavelets and Operators, Cambridge University Press, Cambridge.

[22] Meyer, Y. \& Coifman, R. (1997). Wavelets: Calderón-Zygmund and Multilinear Operators. Cambridge University Press, Cambridge, UK.

[23] Strang, G. (1992). Wavelet and dilation equations: a brief introduction. SIAM Review 31, 614-627.

[24] Tikhonov, A.N. \& Arsenin, V.Y. (1977). Solutions of Ill-posed Problems. Wiley, New York.

[25] Young, R.M. (1976). An introduction to Nonharmonic Fourier Series. Academic Press: New York. 